\title{
Orientation Imaging and Reorientation Dynamics of Single Dye Molecules
}

\author{
Kenneth D. Weston* and Lori S. Goldner \\ The National Institute of Standards and Technology, Gaithersburg, Maryland 20899 \\ Received: April 10, 2000; In Final Form: February 15, 2001
}

\begin{abstract}
We introduce new techniques for obtaining single-molecule orientation images and analyzing the reorientation dynamics of single fluorescent molecules. Polarization modulation is used with confocal microscopy to measure the absorption dipole orientation and reorientation dynamics of individual dye molecules physisorbed to glass and embedded in thin, spin-cast polymer films under ambient conditions. Discrete jumps in absorption dipole orientation are observed for a significant fraction of dye molecules in all samples tested, and in all cases the distribution of first-jump times can be fitted by a stretched exponential function. A sub-population of dye molecules that is stationary on the time scale of these experiments $(32 \mathrm{~s})$ is observed and persists even at high excitation power. Data analysis techniques are developed for quantifying the reorientation dynamics of single molecules, and a novel method for quickly identifying rotationally mobile molecules in a scan field is introduced. A dependence of the reorientation dynamics on film thickness is identified: $\mathrm{DiIC}_{18}$ molecules reorient with higher frequency and a broader distribution of jump rates in progressively thinner polymer films.
\end{abstract}

\section{Introduction}

The extension of fluorescence spectroscopy to the singlemolecule (SM) regime has allowed access to a wide range of phenomena that are obscured in ensemble measurements. ${ }^{1-4}$ For example, we now know that the properties of partially immobilized SMs such as fluorescence intensity, ${ }^{5-7}$ spectral shape, ${ }^{7}$ spectral position, ${ }^{8}$ fluorescence lifetime, ${ }^{9}$ triplet lifetime, ${ }^{10,11}$ and intersystem crossing rate $^{11}$ can fluctuate in time indicating changes in the conformation of the fluorophore or the chemical or physical environment in which it resides. In addition, the distributions of these characteristics can be used to identify and study sub-populations. ${ }^{7,12-14}$ SM fluorescence detection techniques also offer new means of measuring translational motion and diffusion, either by tracking the path of a SM through a medium ${ }^{15-17}$ or by measuring the length of time a molecule spends in a detection volume defined by a focused excitation laser spot. ${ }^{18,19}$ Here we discuss another observable, the dipole orientation of single fluorophores. ${ }^{20-30}$

The ability to query and monitor molecular orientation has significant impact on scientific discovery in biology, chemistry, and physics. ${ }^{31-33}$ In biology, the ability to observe and understand protein folding ${ }^{32}$ and the macromolecular motion of protein catalysts, ${ }^{34,35}$ complexes, ${ }^{36}$ channels, ${ }^{2}$ and motors ${ }^{16,32,33}$ impacts drug discovery and disease treatment. Substantial effort has been expended to understand rotational mobility of proteins and lipids in cell membranes and in cellular function. Novel SM methods of measuring rotational mobility in biological and biomimetic membranes are currently serving to expedite these endeavors. ${ }^{20}$ In chemistry, studies of binding and catalysis that use the efficiency of fluorescence resonance energy transfer (FRET) as a proximity probe ${ }^{2,37,38}$ can be improved when combined with high precision orientation information, since the FRET efficiency depends on relative orientations of donor and acceptor chromophores. In physics, SM orientation monitoring is attractive for understanding

* Author to whom correspondence should be addressed. molecular scale motions and site heterogeneity in polymeric and self-assembled systems. One technologically important example is the development of chromophore-doped polymers as inexpensive alternatives to inorganic nonlinear optical (NLO) materials. ${ }^{39}$ Presently, the temporal decay in alignment of poled chromophores in polymers has prevented widespread application of polymer NLO materials. ${ }^{40}$ Another topic of intense research involves understanding how and why polymer properties in thin films differ from properties in the bulk. ${ }^{41-44}$ These research fields can benefit from new insight provided by observing the static and dynamic properties of individual chromophores.

In this work, we introduce a method of measuring the orientation and reorientation dynamics of individual fluorophores in thin, spin-cast polymer films or deposited directly onto glass substrates. A technique for mapping the absorption dipole orientation of all molecules within a scan range $(100 \mu \mathrm{m} \times 100 \mu \mathrm{m})$ is demonstrated. Reorientation dynamics are observed by monitoring the dipole orientation for many seconds with time resolution of a few milliseconds. We discuss methods to quantify the rotational mobility of a population of SMs and we find for example that rotationally active dye molecules reorient faster in thinner films. Then we introduce and demonstrate a novel technique for quickly identifying which molecules in a scan field are rotationally active.

\section{Experimental Section}

Cover glass substrates are cleaned by plasma etching in room air (Anatech LTD model SP 100). Films containing wellseparated dye molecules are prepared by depositing $10 \mu \mathrm{L}$ of a polymer and dye in chloroform solution onto substrates spinning at $3000 \mathrm{rpm}$. Polymers used were poly(vinyl butyral) (PVB), molecular weight ${ }^{45} M_{W}=115000$ (Scientific Polymer Products Inc.); polystyrene (PS), $M_{W}=500000$ and $M_{W}=1800000$ (Aldrich Chemical Corp.); and poly(2,6-dimethyl-1,4-phenylene oxide) (PPO) with $M_{W}=50000$ (Polysciences, Inc.). The glass transition temperatures, $T_{\mathrm{g}}$, are $51{ }^{\circ} \mathrm{C}, 100{ }^{\circ} \mathrm{C}, 100{ }^{\circ} \mathrm{C}$, and 209 ${ }^{\circ} \mathrm{C}$, respectively. Dyes used were $\operatorname{DiIC}_{18}\left(1,1^{\prime}\right.$-dioctadecyl- 
3,3,3', $3^{\prime}$ - tetramethylindocarbocyanine perchlorate), DiIC 1 (1,1',3,3,3',3'-hexamethylindocarbocyanine iodide), and "Alexa Fluor 532 C5 maleimide" (Molecular Probes). Film thickness is controlled by varying the concentration of the polymer solution. Polymer solution concentrations were $1 \mathrm{mg} / \mathrm{mL}$ and dye concentrations were $0.1 \mathrm{nM}$ except where stated otherwise.

The $\lambda=532 \mathrm{~nm}$ beam of a doubled Nd:YVO 4 laser (QED, GLB series) is passed through a laser band-pass filter, polarizing beam splitter, electrooptic modulator (EOM) (Gsanger model LM0202IR5W), and quarter-wave plate (QWP). Two dielectric mirrors are used to direct the beam through a beam expander and into the rear port of an inverted microscope (Zeiss Axiovert). The EOM $\left(45^{\circ}\right)$ and QWP (vertical $=0^{\circ}$ ) are oriented with respect to the laser polarization (vertical) so that linearly polarized light oriented at an angle proportional to the voltage applied to the EOM is generated. All data discussed here were taken with laser input power of $1 \mu \mathrm{W}$ unless otherwise stated. A dichroic beam splitter (Chroma Technology 545 DCLP) in the microscope reflects the laser beam that is then focused (to $\approx 500 \mathrm{~nm}$ in diameter, limited by diffraction and the quality of the beam spatial mode) on a cover glass surface using a $63 \times$, 1.4 NA oil immersion objective. This objective is also used to collect laser-induced fluorescence; most of the red-shifted emission passes through the dichroic beam splitter $(>90 \%$ transmissivity for $\lambda=550-720 \mathrm{~nm}$ ). A holographic notch filter is used at the detector to further reduce background excitation light. The $150 \mu \mathrm{m}$ diameter active area of a silicon avalanche photodiode (APD) photon counting module (EG\&G SPCM AQR-14) is positioned in the microscope image plane. An X-Y stage with piezo-electric actuators (Polytec PI model P-762.2L) scans the sample. The stage position is controlled using two digital-to-analog output channels of a computer interface board (National Instruments PCI-6110E) and Labview software. The same board counts pulses from the APD.

For orientation measurements, the EOM was driven by a 125 $\mathrm{Hz}$ ramp waveform $(-190 \mathrm{~V}$ to $+190 \mathrm{~V})$ through retardance from $-\lambda / 2$ to $\lambda / 2$. With the QWP as described above, linearly polarized light with polarization angle rotating in time through $180^{\circ}$ is generated. This condition is verified by placing a polarizer and detector in the beam before entrance to the microscope, or in the sample plane, and observing the shape and amplitude of the intensity modulation for all polarizer orientations. In both cases a sine-squared modulation is observed at all orientations with amplitudes that vary no more than 5\% from maximum and offsets that are negligible, as expected for rotating linearly polarized light. The phase of the modulated fluorescence with respect to that of the ramp waveform used to drive the EOM indicates the orientation of the absorption dipole of the molecule. This imaging technique is conceptually analogous to that reported in ref 46 used to spatially resolve absorption dichroism in mesostructured materials. Higgins et al. used lock-in detection to measure the amplitude and phase of rotating linearly polarized light modulated in intensity by the anisotropic absorption of a sample. T. Ha et al. used polarization modulation to measure the orientation of SMs on a somewhat slower time scale than that presented here. ${ }^{21}$ Orientation imaging of SMs using this method has not been demonstrated prior to this work.

To determine orientation, the fluorescence measurement must be synchronized with the modulation phase of the excitation light (i.e., the ramp waveform used to drive the EOM). We use a timing pulse at the start of photon counting (a buffered acquisition of 32 samples at $1 \mathrm{~ms}$ intervals) to trigger a function generator (in burst mode) and amplifier which supply the ramp waveform. Four cycles of $125 \mathrm{~Hz}$ waveform are generated with the same phase at the start of each data acquisition sequence. There is a lag time of 1 to $5 \mathrm{~ms}$ between $32 \mathrm{~ms}$ acquisition periods during which data are stored and the stage is repositioned. During imaging, a line of data are processed [modulation phase and amplitude are calculated using a Fast Fourier Transform (FFT)], displayed, and stored to disk with a dead time of $\approx 30 \mathrm{~ms}$ at the end of each line. No data are acquired during retrace.

To monitor SM orientation continuously over extended periods (typically $32 \mathrm{~s}$; many minutes is possible) the confocal spot is centered over a molecule using coordinates from a previously acquired image. The ramp waveform is triggered by the timing pulse, as above. We measure SM fluorescence continuously over 4000 cycles. Counts are recorded with $1 \mathrm{~ms}$ integration time for 32000 data points (32 s total observation time). Although orientation can be obtained in less time, we treat 32 consecutive bins as one orientation measurement to improve precision. To measure the orientation trajectory of a molecule at two different excitation powers, a liquid crystal modulator (Thorlabs, CR200) is placed between the laser output and the EOM. The excitation power is controlled with a digitalto-analog output from the acquisition computer.

We discuss only orientation in the $(x-y)$ sample plane and make no attempt to measure orientation in $z$ (the optical axis of the system). Several recent papers discuss techniques for measuring the $z$ component of the absorption dipole..$^{23,26-28}$

\section{Results and Discussion}

Orientation imaging of a field of DiIC $_{18}$ SMs dispersed in a $60 \mathrm{~nm} \pm 20 \mathrm{~nm}$ (estimated standard deviation) thick film of PS is demonstrated in Figures 1 and 2. The data for a single pixel in a 200 pixel $\times 200$ pixel image $(20 \mu \mathrm{m} \times 20 \mu \mathrm{m})$ consists of 32 points taken at $1 \mathrm{~ms}$ intervals (Figure 1a). Peaks in the fluorescence detected from a SM result as the linearly polarized excitation light aligns with the molecule's absorption dipole axis. When the excitation polarization is perpendicular to the absorption dipole, the signal is equal to or very near the background, as expected for a single, stationary dipole. The phase of the signal indicates the orientation, $\psi$, of the dipole $(\psi$ $=\varphi / 2$, where $\varphi$ is the phase of the FFT at $125 \mathrm{~Hz}$ ). The data set for one line in an image (Figure 2) is shown in Figure 1b. Along the ordinate are plotted the 32 data points taken at each pixel. The abscissa coordinate is pixel number. There are 200 pixels $(20 \mu \mathrm{m})$ comprising one image line. Several molecules across this line give rise to horizontally striped regions. The orientations of these molecules are related to the vertical position of the stripes. The data set at each pixel is used to generate the plots in Figure 1c-1e. The average count rate for each pixel is shown in Figure 1c. The FFT of each 32 point data set (4 cycles) is calculated and the component at $125 \mathrm{~Hz}$ is used to determine the modulation amplitude $\mathrm{A}$ and orientation $\psi$ at each pixel (Figure 1d,e, respectively). The data could just as well be fit to a sinusoidal function to obtain $\mathrm{A}$ and $\psi$ (see below) but the FFT method is faster and thus more appropriate for real-time display of the orientation image data. The modulation amplitude follows the average count rate except for rare instances when SMs are not well separated. When Gaussian profiles for molecules oriented in nonparallel directions overlap, we expect an increase in the signal offset and an altered modulation amplitude. The orientation data plotted in black in Figure 1e correspond to the positions of molecules (bright regions in Figure 1b). For pixels where there is no dye molecule, only background signal is detected, and the orientation data are 


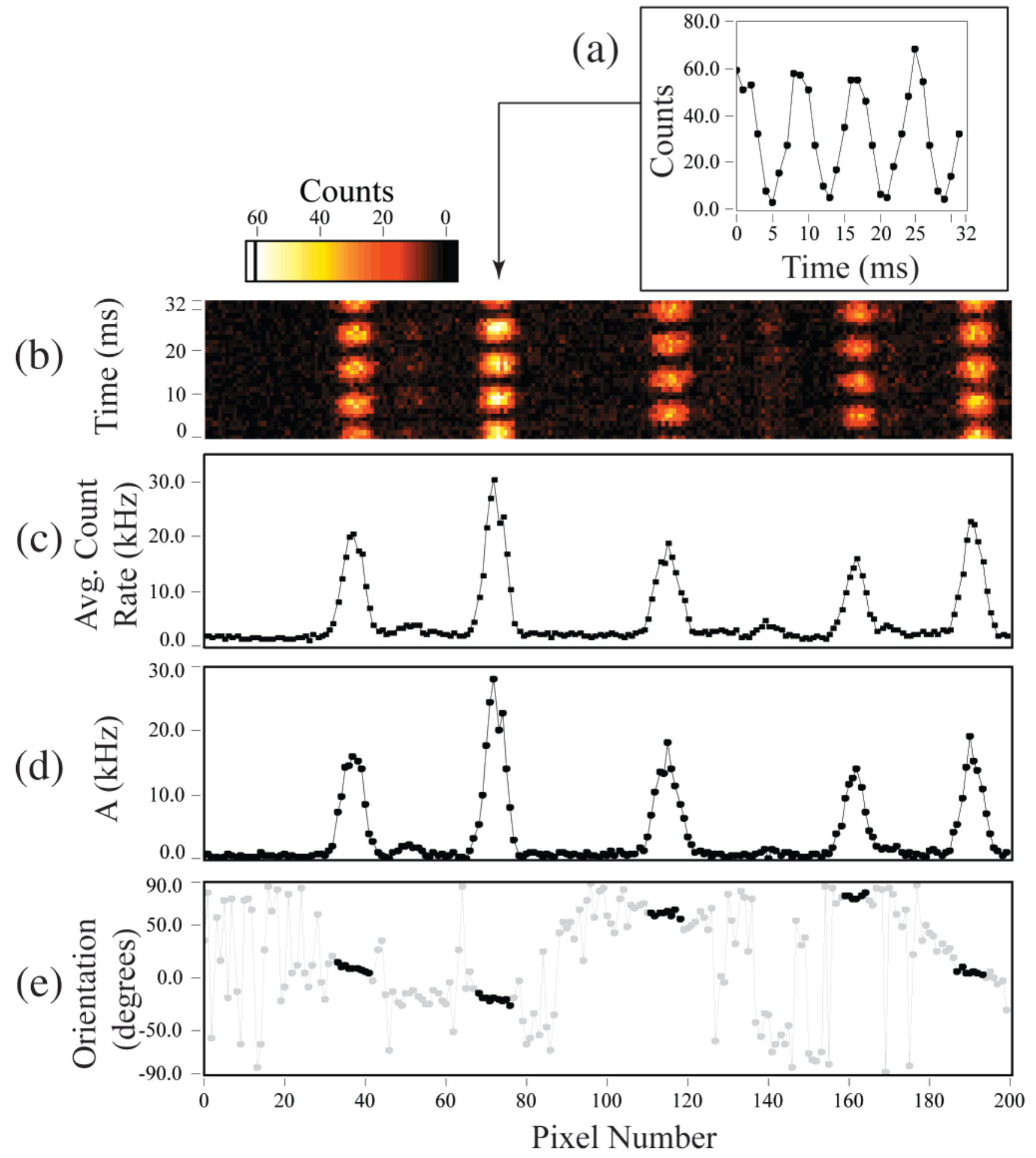

Figure 1. Demonstration of orientation imaging. (a) The modulated fluorescence intensity at one pixel centered over a single DiIC 18 molecule. (b) The modulated fluorescence intensity for one row in an image; the data from each image pixel is displayed in one vertical column. Alternately bright and dark bands represent 5 different molecules, and the shift in the phase of the modulation due to the unique orientation of each molecule is apparent. (c) The averaged fluorescence count rate for each column in (b). (d,e) The modulation amplitude A and orientation for each column in (b).

scattered over the entire scale (points plotted in gray have corresponding fluorescence count rates below $5 \mathrm{kHz}$ ).

Complete images showing average count rate and modulation phase for a $20 \mu \mathrm{m} \times 20 \mu \mathrm{m}$ area of DiIC $_{18}$ molecules in PVB are shown in Figure 2a,b. Solid color regions in the phase image correspond to locations of single dye molecules with welldefined orientation. At image locations where only backgroundlevel fluorescence is detected, orientation is undefined, and fullscale noise results. In Figure 2c, orientation data corresponding to an average count rate less than $7 \mathrm{kHz}$ are blacked-out for clarity. Molecules that reorient during the image acquisition are evident, i.e., the molecule at the top right corner in Figure 2c.

Other groups have demonstrated other molecular dipole orientation mapping techniques. ${ }^{23,26,28,47,48}$ Several features of our method are the high precision [a precision better than $2^{\circ}$ (standard deviation) is typical - see below], millisecond time resolution, insensitivity of the orientation measurement to changes in fluorescence intensity from causes other than an 

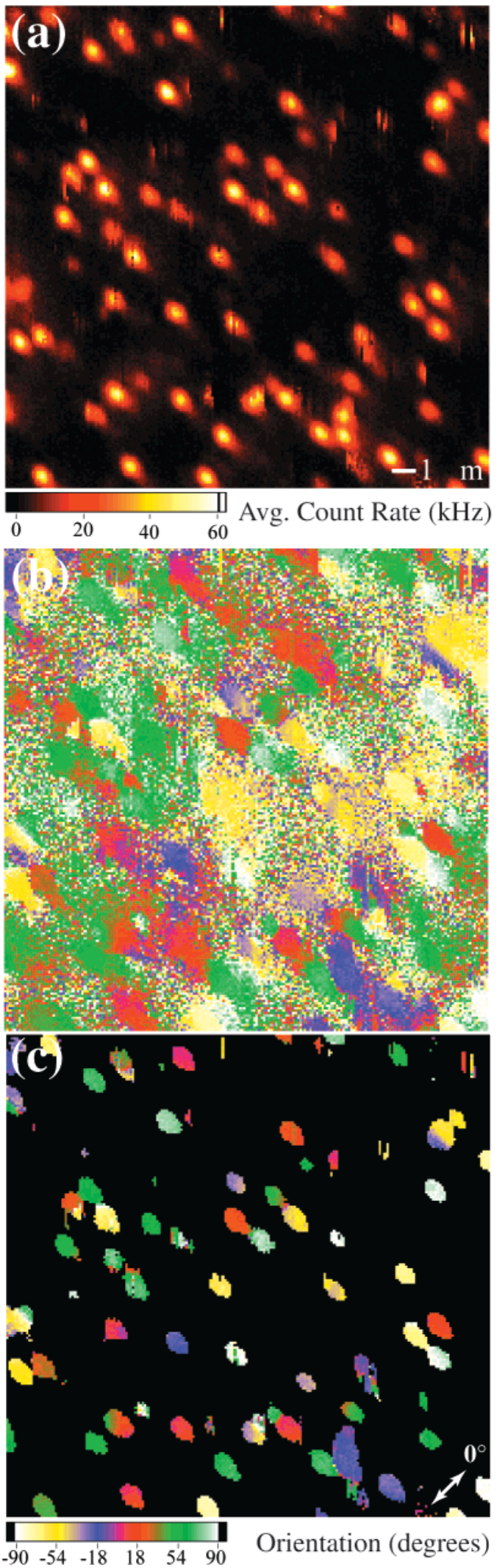

Figure 2. Fluorescence and orientation images of single $\mathrm{DiIC}_{18}$ molecules in a thin PVB film. (a) The average fluorescence count rate. (b) The orientation as deduced from the phase of the modulated fluorescence. (c) The same as (b), except that regions with fluorescence signal below $7 \mathrm{kHz}$ have been excluded from the color scale (appear black).

orientation change, and the ability to distinguish a SM from two or more closely spaced (nonparallel) molecules. In addition, only one detector is needed-to study dipole reorientation dynamics we monitor the orientation of SMs continuously over time. Examples for DiIC ${ }_{18}$ molecules in a $60 \mathrm{~nm} \pm 20 \mathrm{~nm}$ PVB film are shown in Figure 3. Figure $3 a-d$ shows the periodic fluorescence intensity signal from four different molecules illuminated under the same conditions discussed above, with data corresponding to four cycles of polarization modulation shown in each column. For these data, there is no time gap between consecutively acquired orientation measurements; the choice of four cycles per column in the display and analysis is arbitrary. The average count rate (polarization averaged) and orientation of the molecule are plotted below the raw data; here, however, the orientation is obtained by a fit of the data to

$$
\text { fluorescence signal }=A \sin (2 \pi f t+\phi)+c
$$

where $A$, the modulation amplitude, $c$, the offset, and $\phi$, the phase, are fit parameters; $f$ is the modulation frequency; and $t$ is the independent variable corresponding to time (seconds). The phase $\phi$ is related to the orientation $\psi$ by $\psi=\phi / 2$. The advantage of fitting the data over using a FFT is the ability to obtain $c$ and the uncertainty in $\phi$. The standard deviation in $\phi$ is typically $\sigma=3.5^{\circ}$ for a molecule with an average count rate of $27 \mathrm{kHz}$. Both are used in an algorithm for identifying reorientation events for each molecule (see below). The data in Figure $3 \mathrm{a}$ illustrate a molecule with no measurable change in orientation, as evidenced by the parallel horizontal stripes in the raw data and the constant calculated phase. After an irreversible photobleaching event the signal intensity drops to the background and the phase is no longer meaningful. The data in Figure $3 b$ show a molecule that resides in three different orientations. Note that on the orientation scale $-90^{\circ}$ is the same as $90^{\circ}$. In the first $3 \mathrm{~s}$ of data in Figure $3 \mathrm{~b}, \psi$ is very close to $90^{\circ}$ and noise causes the measurement to jump between $90^{\circ}$ and $-90^{\circ}$ (such jumps are excluded from the statistics reported below). At about $4 \mathrm{~s}$ there is a very small change in orientation to just above $-90^{\circ}$. At $12 \mathrm{~s}$ the first of 8 transitions to a shortlived orientation near $20^{\circ}$ takes place. For these data, changes in the average count rate are correlated with changes in $\psi$. This intensity change is likely due to reorientation of the absorption dipole into closer alignment with the $x-y$ plane where absorption is more efficient. However, other photophysical properties may be affected by the new configuration of the SM such as the fluorescence or triplet lifetimes, intersystem crossing yield, or emission spectrum. ${ }^{5-11}$ The next two examples, Figure $3 \mathrm{c}$ and Figure 3d, show progressively more SM rotational dynamics. Figure $3 \mathrm{c}$ demonstrates a molecule that exhibits many different orientations, with sudden, often reversible transitions between them. Again, average count rate changes are correlated with orientation jumps. Figure $3 \mathrm{~d}$ shows a SM that exhibits stable orientation states separated by regions of rapid orientation fluctuations that are too fast to resolve here. Within signal-tonoise limitations, it is possible to observe more frequent orientation jumps by increasing $f$ and shortening the sampling time.

A typical $\sigma=3.5^{\circ}$ results in a precision in $\psi$ of $1.8^{\circ}$. Several other factors also contribute to the overall uncertainty in $\psi$. These primarily involve the degree to which the polarization state of the excitation light is known at the SM. The 5\% modulation in the polarization state of the light discussed in the Experimental section above corresponds to a maximum uncertainty of $3^{\circ}$ in $\varphi$. Additional uncertainty arises from small differences in the location of molecules in the Gaussian spot. Excluding this last factor, we estimate the total uncertainty in $\varphi$ to be $4.6^{\circ}$, or $2.3^{\circ}$ in $\psi$. 

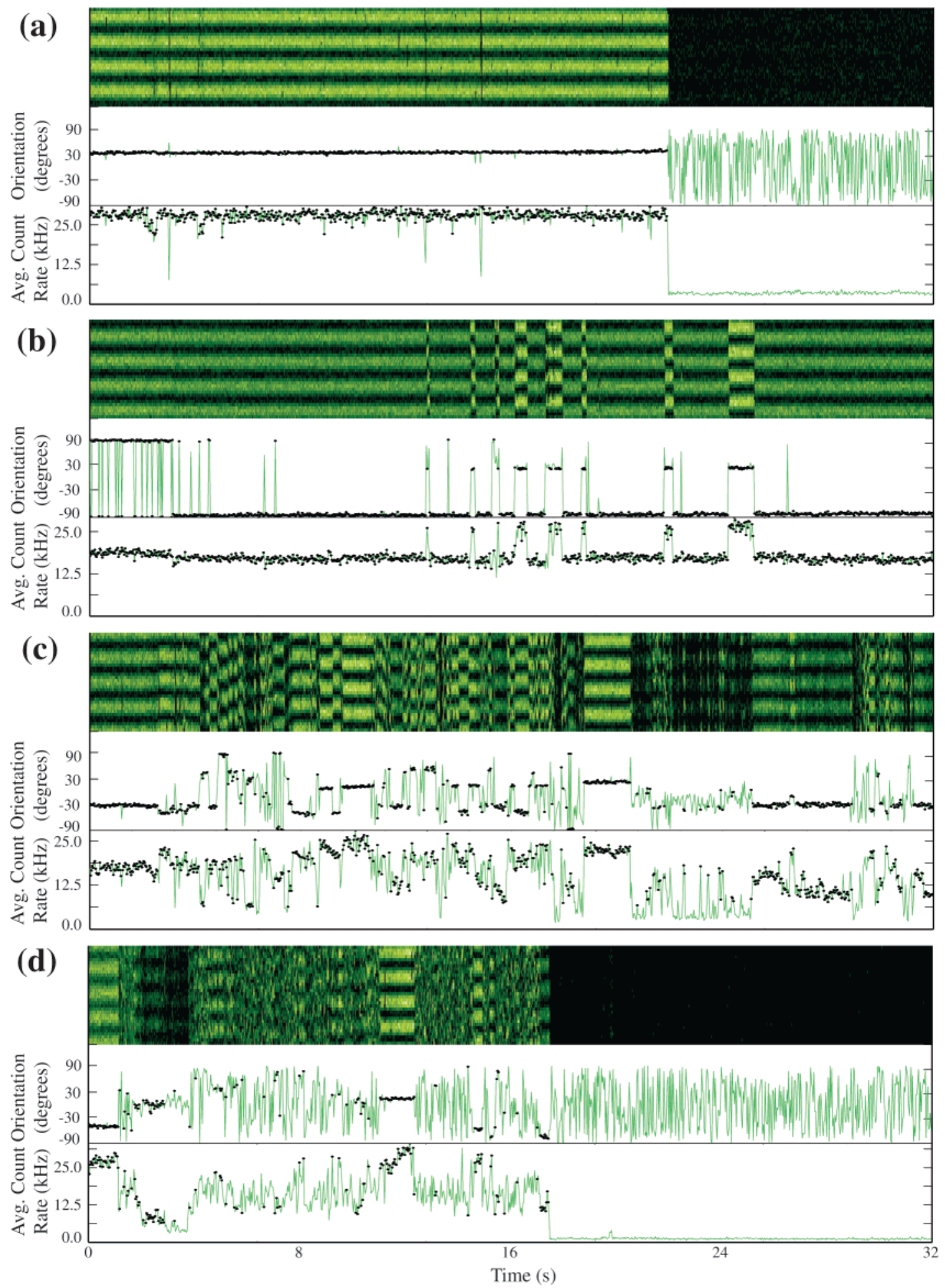

Figure 3. Representative examples of single molecule reorientation dynamics. The top panel in $(\mathrm{a}-\mathrm{d})$ shows the fluorescence intensity data from four $\mathrm{DiIC}_{18}$ molecules excited with rotating linearly polarized light. Each column displays $32 \mathrm{~ms}$ (4 modulation cycles) worth of data. The phase of the modulated fluorescence gives the orientation. The orientation and average count rate are plotted below each intensity panel.

To quantify the reorientation dynamics of these molecules we plot histograms of the observed rotational jump frequencies in Figure 4. To make these histograms, we use the fits of eq 1 to 32-point data segments as described above. Changes in the fluorescence intensity from a SM over time can complicate this task, as do rotational dynamics faster than $32 \mathrm{~ms}$. Data segments are eliminated from the analysis if the integrated number of counts drops below a threshold of 200 per $32 \mathrm{~ms}$, or the modulation amplitude is less than 2.5 counts. Except where a second molecule is present, the modulation amplitude $(A)$ should always equal the offset (c); if this is not the case, it is considered evidence that a second molecule may be present. Therefore only data where $c$ is within $30 \%$ of $A$ are used. Fits for which $\chi^{2}$ was greater than 3 (a very poor fit) generally indicated reorientation(s) occurred during the $32 \mathrm{~ms}$ segment; these segments are also discarded from the analysis. The data included in the analysis are indicated by dark points in the amplitude and orientation plots of Figure 3. We consider that an orientation jump event has occurred when consecutive phase measurements differ by more than $6 \sigma$. Note that this routine counts only jumps 

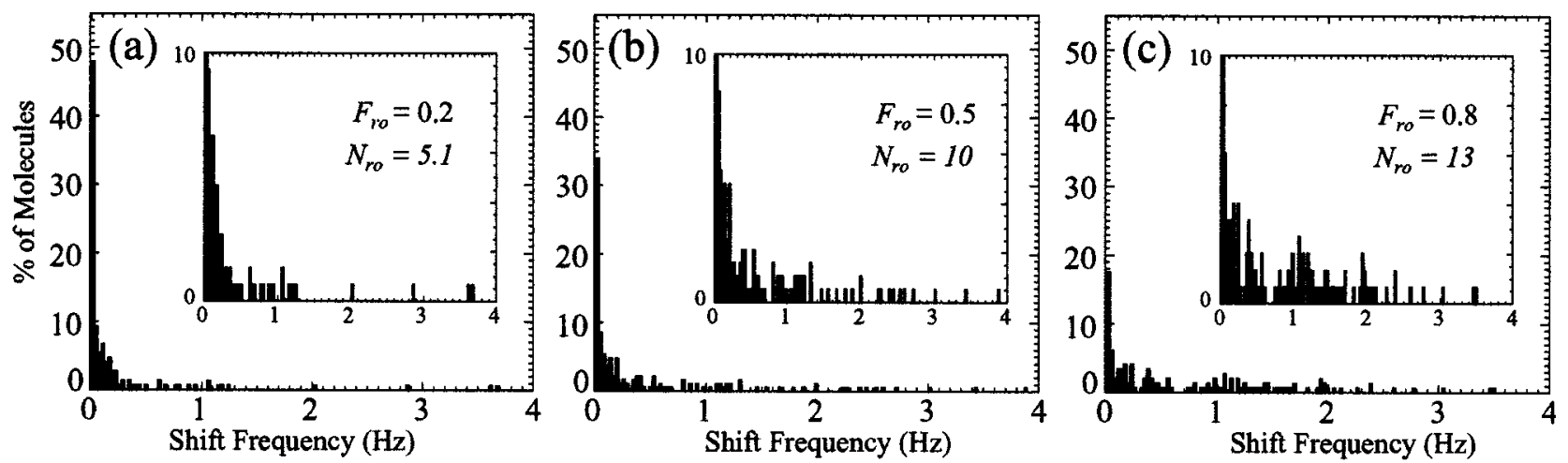

Figure 4. Histograms of the reorientation frequency for populations of molecules under different conditions. The insets for each show the same data with an expanded ordinate (\% of molecules). (a-c) DiIC ${ }_{18}$ molecules embedded in progressively thinner PVB films. (a) PVB film $60 \mathrm{~nm} \pm$ $20 \mathrm{~nm}$ thick. (b) PVB film less than $10 \mathrm{~nm}$ thick. (c) No PVB. The average shift frequency, $F_{\text {ro }}$, and the average number of shifts per molecule, $N_{\text {ro, }}$, are given.

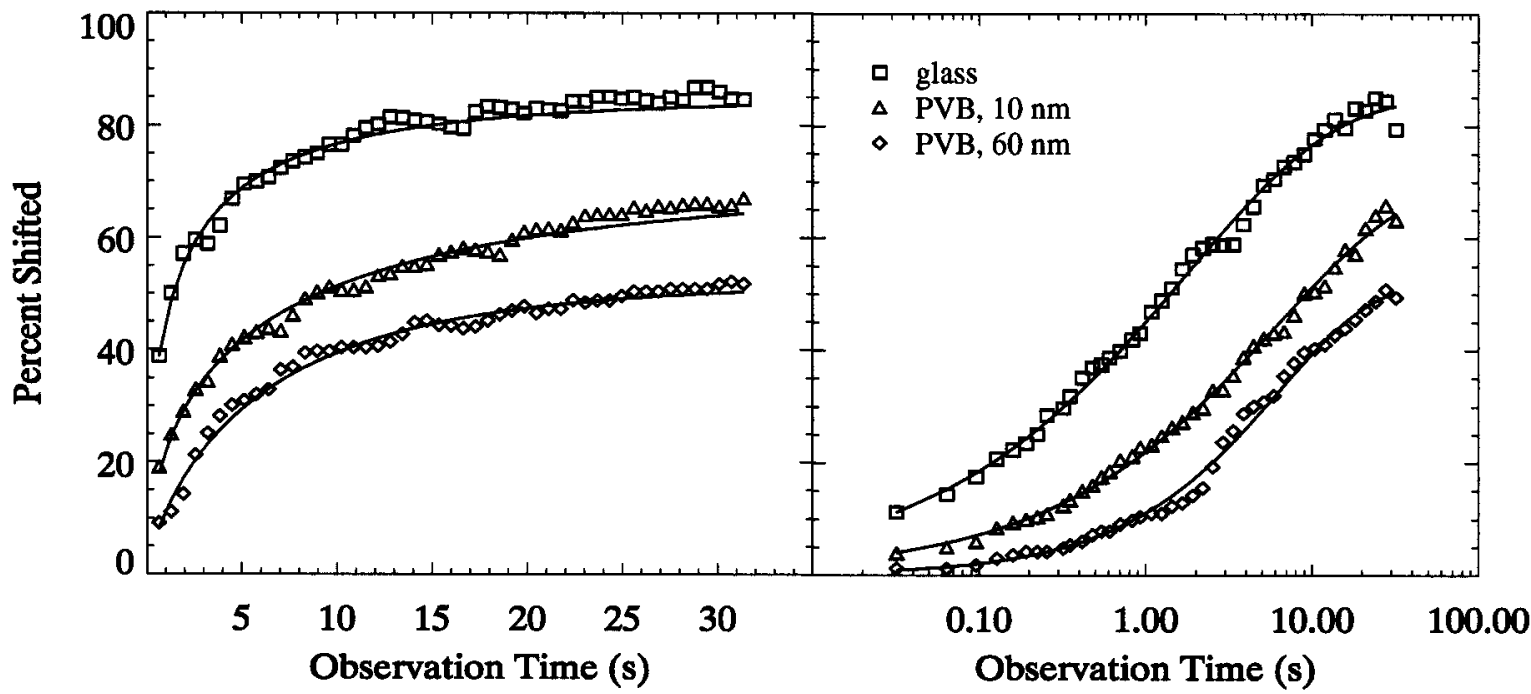

Figure 5. Plots showing the percentage of molecules that reoriented (at least once) as a function of the observation time and the corresponding fits (solid lines) of a stretched exponential function to the logarithmically spaced data (right side). The same fits are shown again against the linearly spaced data (left side). The samples are the same as those used in Figure 4. Diamonds: DiIC18 in PVB, $60 \mathrm{~nm}$ thick. The fit parameters are $\tau=$ $198 \pm 55, \alpha=0.78 \pm 0.07$, and $A_{\text {mobile }}=52 \pm 5$. Triangles: DiIC18 in PVB, less than $10 \mathrm{~nm}$ thick. The fit parameters are $\tau=194 \pm 96, \alpha=$ $0.54 \pm 0.05$, and $A_{\text {mobile }}=71 \pm 10$. Squares: DiIC18 on glass. The fit parameters are $\tau=55 \pm 16, \alpha=0.49 \pm 0.05$, and $A_{\text {mobile }}=85 \pm 5$.

between quasi-stable configurations and cannot account for the rotation of molecules on a time scale faster than $32 \mathrm{~ms}$ (i.e., it must be possible get a good fit in each $32 \mathrm{~ms}$ segment or the data are not used).

Figure 4 shows histograms of the frequency of reorientation events for DiIC $_{18}$ molecules under three different PVB thickness conditions. Because many molecules bleach before the end of the observation time, the shift frequency (total number of shifts over total lifetime of the molecule) is more meaningful than number of shifts. To highlight the smaller percentage contributions at higher frequencies, the data are re-plotted with an expanded $y$-axis in the inset of each figure.

The reorientation frequency histogram in Figure $4 \mathrm{a}$ is from data acquired on a sample spin cast from a $1 \mathrm{mg} / \mathrm{mL}$ PVB, 0.1 $\mathrm{nM} \mathrm{DiIC}_{18}$ solution. The polymer film is $60 \mathrm{~nm} \pm 20 \mathrm{~nm}$ thick based on spectroscopic reflectometry measurements of similarly prepared films on a silicon substrate. Of 150 molecules, 72 (48\%) were rotationally immobile (the zero frequency peak) while the other $52 \%$ reoriented one or more times. The average reorientation frequency, $F_{\text {ro }}$, for the data in Figure $4 \mathrm{a}$ is 0.21 $\mathrm{Hz}$, although the standard deviation of this distribution is larger than the average (approximately $0.5 \mathrm{~Hz}$ ) and it is clear more data are needed if we want to study the ensemble behavior in this way. Figure $4 \mathrm{~b}$ shows the histogram for a sample spin cast from a $0.1 \mathrm{mg} / \mathrm{mL}$ PVB, $0.1 \mathrm{nM} \mathrm{DiIC}_{18}$ solution. Based on the 10-fold decrease in PVB concentration and thickness of $1 \mathrm{mg} /$ $\mathrm{mL}$ PVB films, this film is less than $10 \mathrm{~nm}$ thick. For this sample, 64 of the 188 molecules $(34 \%)$ did not reorient, somewhat less than for the thicker film. In addition, a noticeably larger proportion of molecules displayed reorientation frequencies above $0.5 \mathrm{~Hz}$. For Figure $4 \mathrm{~b}, F_{\text {ro }}=0.45 \mathrm{~Hz} \pm 0.8 \mathrm{~Hz}$. The trend continues in Figure $4 \mathrm{c}$ which shows the reorientation frequency histogram for a sample with no PVB included in the spin cast solution. For DiIC 18 molecules directly on plasmaetched glass, only 26 of 148 molecules (18\%) are stationary and still more molecules have high reorientation frequencies $\left(F_{\text {ro }}=0.80 \mathrm{~Hz} \pm 0.8\right)$.

A similar trend can be seen if we plot the percent of molecules that have reoriented at least once as a function of observation time (Figure 5). The same data sets used to generate the histograms in Figure 4 are used. Molecules which photobleach during the $32 \mathrm{~s}$ observation are excluded from the percentage calculations after the time at which they bleached. The plots for all three samples are shown in Figure 5. Data points evenly spaced on a linear scale are shown on the left; data points evenly spaced on a logarithmic scale are shown on the right (except at 
small times where the points are sparse). Solid lines are bestfits of a stretched exponential function, $f(t)=A_{\text {mobile }} \times(1-$ $\left.\exp \left[-(t / \tau)^{\alpha}\right]\right)$, to the points shown on the right, where the fit parameters $A_{\text {mobile, }} \tau$, and $\alpha$ are, respectively, the fraction of the molecules that is mobile, the relaxation time, and an exponent that reflects the distribution of relaxation times in the system (see figure caption for fit results). Fits of a single exponential function (2 adjustable parameters), which would indicate simple two-state relaxation, generally fit very poorly. Fits of a double exponential function (4 parameters) are often good but for the examples used here did not fit as well as the stretched exponential. Fits with $A_{\text {mobile }}$ fixed and equal to 1 were worse, especially for thick film samples where the fraction of molecules reorienting in $32 \mathrm{~s}$ was smaller. Data over many more decades in time is required to clearly differentiate between the various forms, and sample-to-sample variations of our as-cast samples make quantitative interpretation difficult. A stretched exponential form is consistent in general with relaxation in polymers or glassy materials, where excitations of arbitrarily low energy are present and relaxation rates may be a function of time. ${ }^{49}$ However, from Figure 5 we cannot rule out that this stretched exponential form is the result of a distribution of relaxation times due to static disorder in the system and not a result of a time-dependent relaxation rate. This is likely the case for molecules on a bare glass surface. For molecules embedded in polymers, previous work on the relaxation of dye molecules in poled polymers shows clear evidence of stretched exponential relaxation. What is clear from Figure 5 is that the glass surface has overall faster dynamics and more molecules participating in the dynamics than either polymer surface.

Surface effects may contribute to the difference between thin and thick samples. A number of studies have found that polymer mobility near an interface can vary significantly from the properties in the bulk. ${ }^{42-44,50}$ Thinner films are expected to have a larger proportion of dopant dye molecules residing at the polymer-air and polymer-glass interfaces. This is a possible explanation for the trend of results shown in Figures 4 and 5; the rotationally mobile molecules could all be at one or the other interface while the rotationally stable ones are in the bulk. To test this idea, the following experimental scheme was used. First, polymer-dye films on gold-coated glass substrates were made. A $5 \mathrm{~nm}$ thick gold film was deposited on cleaned cover glass using magnetron sputtering. The gold quenches fluorescence from molecules near the surface ${ }^{51}$ so that all SMs detected from this sample are either in the bulk or at the polymer-air interface. Fluorescence quenching due to the gold was verified by the absence of fluorescence from thin dye-doped polymer films (prepared by spin casting $1 \mathrm{mg} / \mathrm{mL}$ PVB, $1 \mathrm{nM} \mathrm{DiIC}_{18}$ at 3000 $\mathrm{rpm})$. In thicker films on gold $\left(20 \mathrm{mg} / \mathrm{mL}\right.$ PVB, $0.1 \mathrm{nM} \mathrm{DiIC}_{18}$ spin cast at $80 \mathrm{rpm}$ ), SM fluorescence could be detected. A study of 217 molecules yielded a histogram similar to that shown in Figure $4 \mathrm{a}$ with $F_{\text {ro }}=0.18 \mathrm{~Hz} \pm 0.4 \mathrm{~Hz}$ and $48 \%$ of molecules stationary. This result rules out the possibility that only dye molecules at the polymer-glass interface are rotationally mobile. To address the possibility that only dye molecules at the polymer-air interface are rotationally mobile we attempted to study molecules in the center of a film many microns thick. This approach is complicated by an increase in fluorescence background and no meaningful data was obtained. As an alternative, a thin overlayer PVB film was deposited on the existing film by spin casting a $1 \mathrm{mg} / \mathrm{mL}$ PVB solution on top while spinning the sample at $3000 \mathrm{rpm}$. Statistics on SM reorientations were acquired (133 molecules). The histogram generated had $F_{\text {ro }}=0.15 \mathrm{~Hz} \pm 0.3 \mathrm{~Hz}$ and $40 \%$ of molecules

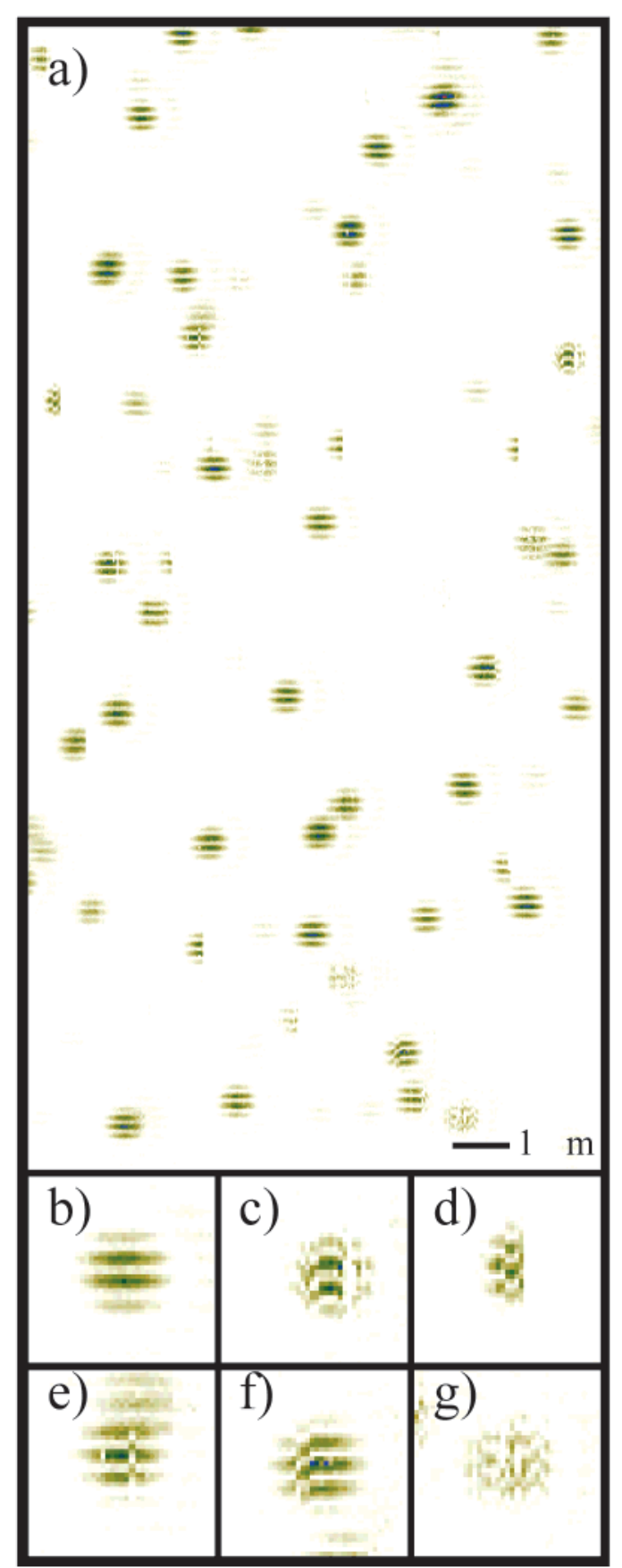

Figure 6. Demonstration of the technique used to quickly assess the location and fraction of rotationally mobile molecules in a sample. The beginning of each scan line (vertical) is synchronized with the initiation of polarization rotation. The bright and dark stripes correspond to excitation light parallel to or perpendicular to the absorption dipole orientation of a molecule. Discontinuity in the stripes indicates a molecule has reoriented during imaging. In (a) a 300 pixel $\times 600$ pixel $(10 \mu \mathrm{m} \times 20 \mu \mathrm{m})$ image is shown. In (b) $-(\mathrm{g})$, expanded views of 6 different molecules taken from the image of (a) are shown. The molecule in (b) is stationary while the molecules in $(\mathrm{c})-(\mathrm{g})$ are rotationally active.

stationary, and was similar to that of Figure 4a. This technique does not entirely rule out a contribution from molecules sitting at the air-polymer interface since fluorescent molecules may diffuse to the surface during spin casting. We nonetheless take these results as evidence that rotationally mobile molecules exist in the bulk of the PVB film and not exclusively at the interfaces. Further evidence for this conclusion comes from the observation that reorientation dynamics were again not obviously altered 

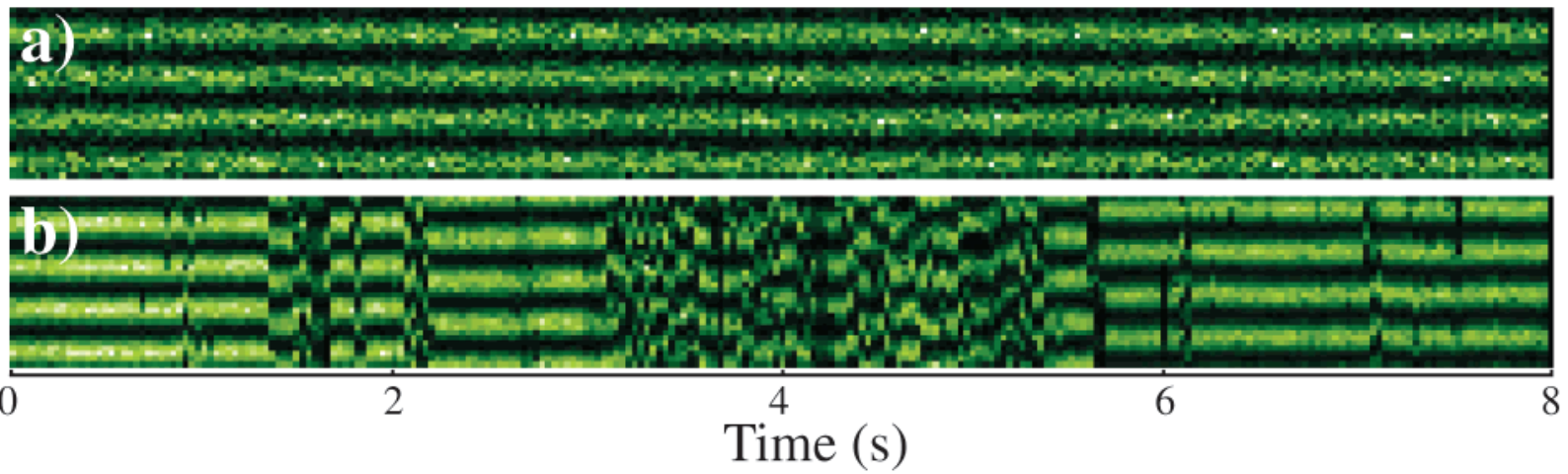

Figure 7. Demonstration of excitation power dependence of orientation jump frequency. Intensity as a function of time data shown in the same format as in Figure 3 for one molecule excited using (a) $2 \mathrm{uW}$ and (b) $20 \mathrm{uW}$ excitation power. The color scale in (a) represents 0 to 24 counts and in (b) 0 to 284 counts.

when a pre-cured poly(dimethylsiloxane) flat was brought into conformal contact with the PVB surface.

Reorientation data for DiIC 18 molecules in films of PS $\left(M_{W}\right.$ $=500000)$, and PPO were also obtained. The glass transition temperature $\left(T_{\mathrm{g}}\right)$ of $\mathrm{PVB}, \mathrm{PS}, \mathrm{PPO}$ are $51^{\circ} \mathrm{C}, 100{ }^{\circ} \mathrm{C}$, and 209 ${ }^{\circ} \mathrm{C}$, respectively. All samples studied were similar in that they had a large fraction of mobile molecules and a broad distribution of jump rates.

In addition to the method demonstrated in Figures 1 and 2 for mapping the orientation of all the individual molecules in a scan field, we devised a polarization modulation technique which allows one to quickly locate molecules that reorient during imaging (Figure 6). In the data of Figure 2c, molecules that reorient during the imaging process can be identified by the sudden change in phase during or between line scans over a single molecule. To find the orientation of the molecule the scan was paused at each pixel for $32 \mathrm{~ms}$ while polarization modulation data was acquired. Changes in orientation were only evident after Fourier analysis of the data at each pixel. In Figure 6 , we quickly identify molecules that are rotationally mobile without pausing to take modulation data at each point. Instead, the beginning of each scan line is synchronized with the initiation of the ramp waveform $(200 \mathrm{~Hz})$ driving the EOM. At each pixel, a single data point with $1 \mathrm{~ms}$ integration time is acquired. Stationary molecules then appear to have "stripes" across them with orientation perpendicular to the scan direction. The bright/dark regions correspond to times at which the polarization of the excitation light is parallel/perpendicular to the absorption dipole moment. Molecules that change orientation show a shift in the position of these stripes. Figure 6a shows a $10 \mu \mathrm{m} \times 20 \mu \mathrm{m}(300$ pixels $\times 600$ pixels $)$ image produced using this method for DiIC 18 in a $60 \mathrm{~nm}$ thick PVB film. The fast scan axis is vertical. Illumination power level was $5 \mu \mathrm{W}$. Note that this technique is also useful for distinguishing between single molecules which have a well-defined absorption dipole axis and clusters of molecules that have only partial or no sensitivity to the polarization of the excitation light. In Figure $6 \mathrm{~b}-\mathrm{g}$, expanded views taken from Figure $6 \mathrm{a}$ are shown. The molecule in Figure 6b, like most in the whole image (83\%), is striped and does not reorient. The molecules shown in Figure $6 c-g$ are examples of molecules which show clear evidence of reorienting during imaging. Seventeen percent of the molecules in this image showed such orientation jumps. It is possible to use the locations of peaks and troughs across a molecule and the known direction of the excitation polarization for each pixel to determine the orientation of a molecule with this method.

The $17 \%$ of molecules that are rotationally mobile in Figure 6 (taken at $5 \mu \mathrm{W}$ ) seems considerably higher than would be predicted by Figure 5, which was from data excited with laser power of $1 \mu \mathrm{W}$. That is, for a laser spot $0.5 \mu \mathrm{m}$ in diameter, each molecule in Figure 6 is illuminated for approximately 180 ms during the scan. At $180 \mathrm{~ms}$, Figure 5 would predict that about $5 \%$ of the molecules have reoriented. Since $17 \%$ of the molecules in Figure 6 reorient, this seems to indicate that the jumps are photoinduced. However if these orientation jumps are not photoinduced, $180 \mathrm{~ms}$ may not be the relevant time scale. Instead the relevant time scale may be the time over which each molecule is visited, or about $4.5 \mathrm{~s}$. At $4.5 \mathrm{~s}$, Figure 5 would predict that $25 \%$ of the molecules have reoriented. Since in either case we cannot rule out sample-to-sample variations to account for these differences, we took images similar to that shown in Figure 6 at different spots on the same sample and powers between 0.5 and $20 \mu \mathrm{W}$. From 1 to $20 \mu \mathrm{W}, 15 \%-20 \%$ of molecules were observed to rotate and no trend was present; at $0.5 \mu \mathrm{W}$ the result was closer to $10 \%$. These results seem to indicate that the process is not photoinduced. In a second set of experiments, we located each of 81 single molecules and monitored them with input laser power of $2 \mu \mathrm{W}$ for $8 \mathrm{~s}$, and then monitored with input power of $20 \mu \mathrm{W}$ for $8 \mathrm{~s}$ (Figure 7). The two alternating power measurements were repeated until each molecule photobleached. Of the molecules studied, 39 showed no orientation jumps at low or high power, 11 molecules photobleached before or within several seconds of the start of the higher excitation power measurement, and 19 molecules had insufficient signal in the low power measurement to see orientation jumps reliably. The data for these molecules $(85 \%)$ is inconclusive for determining whether the reorientation dynamics are photoinduced. However, the other 12 molecules (15\%) showed an obvious increase in reorientation frequency at high power over that at low power. Figure 7 shows one such example. The intensity vs time data in the same format as shown in Figure 3 is shown for a molecule at the low excitation power (Figure 7a) and the same molecule at the higher excitation power (Figure $7 \mathrm{~b}$ ). We also looked for examples where the same or fewer reorientation events occurred at high power than at low power and found none. Were it not for our previous results, we would conclude that the rotational jumps are photoinduced, in agreement with results reported by $\mathrm{T}$. Ha et al. for fluorophores bound to ssDNA and surface bound under aqueous conditions. ${ }^{22}$ Our combined results seem to point to something more subtle; it is possible for example that we are observing the effects of heating (which might, for example, be negligible in the case of Figure 6 but much more important when the laser sits on the same spot for $8 \mathrm{~s}$, as in Figure 7), or that some combination of thermally induced and photoinduced fluctuations are driving these dynamics. 
It has been shown using ensemble techniques that reorientation dynamics of small chromophore probe molecules directly couple to the local segmental motions of a matrix polymer. ${ }^{52}$ It is possible that the reorientation dynamics that we have observed are related to the dynamics of the polymer. Alternately, since we also see reorientation events on the bare glass surface, we are observing the rotational motion of fluorophores in a glassy material, where each fluorophore sits in a different static environment. In either case the stretched exponential form discussed above for rotational dynamics is reasonable. In the future, studies of the time-correlation of the angular motion of these dye molecules with faster time resolution (i.e., so that we have more jumps per molecule before photobleaching) should enable us to use the dynamics of a specific dye molecule as a probe of either local polymer dynamics (if we are observing thermal motion of the polymer) or local structure (if we are observing the motion of dye molecules in an otherwise static environment). Single molecule studies as a function of time and temperature in annealed polymer films should also help to resolve this ambiguity. That we are able to detect clear differences between the reorientation dynamics for individual molecules within a film, and overall differences for molecules in films of varying thickness, is a very promising result.

\section{Conclusions}

We have demonstrated a method for studying the orientation and reorientation dynamics of SMs with a precision of $2^{\circ}$ and accuracy somewhat larger than that. The time resolution of the technique demonstrated here is $32 \mathrm{~ms}$; however, an extension to $4 \mathrm{~ms}$ resolution is immediately possible and faster measurements might be acquired at the expense of overall observation time or measurement precision. The polarization rotation technique was applied in an imaging mode to map the orientation of each molecule in a scanned region. We have initiated application of these techniques to study the dynamics of SMs embedded in thin polymer films. We find differences in the dynamics for molecules embedded in polymer films of different thickness or on glass. In all cases a significant fraction of the molecules is rotationally mobile. In all cases a stretched exponential function can be used to describe the fraction of molecules that has reoriented at least once as a function of time. While some evidence is presented that the motion might be photoinduced, it is clear that it is not strictly photoinduced. Using a new technique that can quickly identify rotationally active molecules, we measured the rotationally active fraction as a function of laser power and found little or no dependence on laser power. This leaves open the possibility that the rotational dynamics are evidence of thermal fluctuations in these polymers, and that the individual fluorophores can be used to study local dynamics in polymers. Earlier work on the reorientation dynamics of dye in a polymer matrix showed that reorientation dynamics were in fact the result of polymer relaxation. ${ }^{39,52}$ Alternately, we are observing the effects of static disorder on dye-molecule dynamics in which case the orientational motion is indicative of local polymer structure.

Acknowledgment. We thank Vitalii Silin and John Elliott for providing the gold-coated cover glass, Jeeseong Hwang for invaluable advice and guidance in microscopy and sample preparation, and Barry Bauer, Joseph Hubbard, and Michael Fasolka for helpful discussions on polymer physics. We are grateful to the NIST ATP focused program in tissue engineering for supporting our work on SM dynamics. Certain commercial equipment, instruments, or materials are identified in this paper to foster understanding. Such identification does not imply recommendations or endorsements by the National Institute of Standards and Technology, nor does it imply that the materials or equipment identified are necessarily the best available for the purpose.

\section{References and Notes}

(1) Moerner, W. E.; Orrit, M. Science 1999, 283, 1670-1676.

(2) Weiss, S. Science 1999, 283, 1676-1683. 480 .

(3) Xie, X. S.; Trautman, J. K. Annu. Rev. Phys. Chem. 1998, 49, 441-

(4) Plakhotnik, T.; Donley, E. A.; Wild, U. P. Annu. Rev. Phys. Chem. 1997, 48, 181-212.

(5) Ha, T.; Enderle, T.; Chemla, D. S.; Selvin, P. R.; Weiss, S. Chem. Phys. Lett. 1997, 271, 1-5.

(6) Dickson, R. M.; Cubitt, A. B.; Tsien, R. Y.; Moerner, W. E. Nature 1997, 388, 355-358.

(7) Weston, K. D.; Carson, P. J.; Metiu, H.; Buratto, S. K. J. Chem. Phys. 1998, 109, 7474-7485.

(8) Lu, H. P.; Xie, X. S. Nature 1997, 385, 143-146.

(9) Ying, L. M.; Xie, X. S. J. Phys. Chem. B 1998, 102, 10399-10409.

(10) Veerman, J. A.; Garcia-Parajo, M. F.; Kuipers, L.; van Hulst, N. F. Phys. Rev. Lett. 1999, 83, 2155-2158.

(11) Weston, K. D.; Carson, P. J.; DeAro, J. A.; Buratto, S. K. Chem. Phys. Lett. 1999, 308, 58-64.

(12) Schaffer, J.; Volkmer, A.; Eggeling, C.; Subramaniam, V.; Striker, G.; Seidel, C. A. M. J. Phys. Chem. A 1999, 103, 331-336.

(13) Ye, J. Y.; Ishikawa, M.; Yogi, O.; Okada, T.; Maruyama, Y. Chem. Phys. Lett. 1998, 288, 885-890.

(14) Macklin, J. J.; Trautman, J. K.; Harris, T. D.; Brus, L. E. Science 1996, 272, 255-258.

(15) Funatsu, T.; Harada, Y.; Tokunaga, M.; Saito, K.; Yanagida, T. Nature 1995, 374, 555-559.

(16) Vale, R. D.; Funatsu, T.; Pierce, D. W.; Romberg, L.; Harada, Y.; Yanagida, T. Nature 1996, 380, 451-453.

(17) Dickson, R. M.; Norris, D. J.; Tzeng, Y. L.; Moerner, W. E. Science 1996, 274, 966-969.

(18) Nie, S. M.; Chiu, D. T.; Zare, R. N. Science 1994, 266, 10181021.

(19) Burden, D. L.; Kasianowicz, J. J. J. Phys. Chem. B 2000, 104, 6103-6107.

(20) Schutz, G. J.; Schindler, H.; Schmidt, T. Opt. Lett. 1997, 22, 651653.

(21) Ha, T.; Enderle, T.; Chemla, D. S.; Selvin, P. R.; Weiss, S. Phys. Rev. Lett. 1996, 77, 3979-3982.

(22) Ha, T.; Glass, J.; Enderle, T.; Chemla, D. S.; Weiss, S. Phys. Rev. Lett. 1998, 80, 2093-2096.

(23) Dickson, R. M.; Norris, D. J.; Moerner, W. E. Phys. Rev. Lett. 1998, 81, 5322-5325.

(24) Ruiter, A. G. T.; Veerman, J. A.; GarciaParajo, M. F.; vanHulst, N. F. J. Phys. Chem. A 1997, 101, 7318-7323.

(25) Ha, T.; Laurence, T. A.; Chemla, D. S.; Weiss, S. J. Phys. Chem. $B$ 1999, 103, 6839-6850.

(26) Jasny, J.; Sepiol, J. Chem. Phys. Lett. 1997, 273, 439-443.

(27) Bopp, M. A.; Jia, Y.; Haran, G.; Morlino, E. A.; Hochstrasser, R. M. Appl. Phys. Lett. 1998, 73, 7-9.

(28) Bartko, A. P.; Dickson, R. M. J. Phys. Chem. B 1999, 103, 30533056.

(29) Hollars, C. W.; Dunn, R. C. J. Chem. Phys. 2000, 112, 7822-7830. (30) Bartko, A. P.; Dickson, R. M. J. Phys. Chem. B 1999, 103, 1123711241

(31) Tanaka, H.; Ishijima, A.; Honda, M.; Saito, K.; Yanagida, T. Biophys. J. 1998, 75, 1886-1894.

(32) Warshaw, D. M.; Hayes, E.; Gaffney, D.; Lauzon, A. M.; Wu, J. R.; Kennedy, G.; Trybus, K.; Lowey, S.; Berger, C. Proc. Natl. Acad. Sci. U.S.A. 1998, 95, 8034-8039.

(33) Sase, I.; Miyata, H.; Ishiwata, S.; Kinosita, K. Proc. Natl. Acad. Sci. U.S.A. 1997, 94, 5646-5650.

(34) Lu, H. P.; Xun, L. Y.; Xie, X. S. Science 1998, 282, 1877-1882.

(35) Ha, T. J.; Ting, A. Y.; Liang, J.; Caldwell, W. B.; Deniz, A. A.; Chemla, D. S.; Schultz, P. G.; Weiss, S. Proc. Natl. Acad. Sci. U.S.A. 1999, 96, 893-898.

(36) Bopp, M. A.; Sytnik, A.; Howard, T. D.; Cogdell, R. J.; Hochstrasser, R. M. Proc. Natl. Acad. Sci. U.S.A. 1999, 96, 11271-11276.

(37) Ha, T.; Enderle, T.; Ogletree, D. F.; Chemla, D. S.; Selvin, P. R.; Weiss, S. Proc. Natl. Acad. Sci. U.S.A. 1996, 93, 6264-6268.

(38) Ha, T. J.; Ting, A. Y.; Liang, J.; Deniz, A. A.; Chemla, D. S.; Schultz, P. G.; Weiss, S. Chem. Phys. 1999, 247, 107-118.

(39) Dhinojwala, A.; Wong, G. K.; Torkelson, J. M. J. Chem. Phys. 1994, 100, 6046-6054. 
(40) Hampsch, H. L.; Yang, J.; Wong, G. K.; Torkelson, J. M. Macromolecules 1990, 23, 3648-3654.

(41) Hall, D. B.; Miller, R. D.; Torkelson, J. M. J. Polym. Sci. Pt. BPolym. Phys. 1997, 35, 2795-2802.

(42) Mounir, E. S. A.; Takahara, A.; Kajiyama, T. Polym. J. 1999, 31, $550-556$.

(43) DeMaggio, G. B.; Frieze, W. E.; Gidley, D. W.; Zhu, M.; Hristov, H. A.; Yee, A. F. Phys. Rev. Lett. 1997, 78, 1524-1527.

(44) Tanaka, K.; Taura, A.; Ge, S. R.; Takahara, A.; Kajiyama, T. Macromolecules 1996, 29, 3040-3042.

(45) According to ISO 31-8, the term "molecular weight" has been replaced with the "relative molecular mass," symbol $M r$. The conventional notation, rather than the ISO notation, has been employed for this publication.
(46) Higgins, D. A.; Reid, P. J.; Barbara, P. F. J. Phys. Chem. 1996, $100,1174-1180$

(47) Betzig, E.; Chichester, R. J. Science 1993, 262, 1422-1425.

(48) Gohde, W.; Fischer, U. C.; Fuchs, H.; Tittel, J.; Basche, T.; Brauchle, C.; Herrmann, A.; Mullen, K. J. Phys. Chem. A 1998, 102, 91099116.

(49) Ngai, K. L. "Evidences for Universal Behaviour of Condensed Matter at Low Frequencies/Long Times". In Non-Debye relaxation in condensed matter; Ramakrishnan, T. V., Lakshmi, M. R., Eds.; World Scientific Publishing Company Inc.: Teaneck, NJ, 1987; pp 23-192.

(50) Frank, B.; Gast, A. P.; Russell, T. P.; Brown, H. R.; Hawker, C. Macromolecules 1996, 29, 6531-6534.

(51) Drexhage, K. H. Interaction of Light With Monomolecular Dye Layers. In Progress in Optics; Wolf, E., Ed.; North-Holland: Amsterdam, 1974; Vol. 12, pp 163-232.

(52) Dhinojwala, A.; Wong, G. K.; Torkelson, J. M. Macromolecules 1993, 26, 5943-5953. 